\title{
Programmable Energy Meter with Bill Estimation for Reducing Power Bill
}

\author{
Gurram Dheeraj Reddy ${ }^{\# 1}$, Gaggalapally Kalyani ${ }^{\# 2}$,Gande Sai Ganesh ${ }^{\# 3}$, G. Anitha Chowdary ${ }^{\# 4}$ \\ $\# 1,2,3$ Under Graduate Students, ECE Department, \\ \#4 Associate Professor, ECE Department \\ TKR College Of Engineering And Technology, Meerpet, Saroornagar, Hyderabad
}

\begin{abstract}
Electricity Energy Meter is a good concept in which you can recharge its balance, like we do in our mobile phones. In this project we are building an automated system by using 8051 microcontroller and GSM module. The main objective of the project is to develop an energy meter that informs the consumer the exact consumption and billing that the load consumes through SMS. For this project, we have taken a standard electronic energy meter. The blinking LED as seen on the front panel is directly proportional to the power consumed. More the power drawn faster becomes the LED blinking pulses. One sensor is placed above such blinking LED to derive the real time units consumed whose output goes to an 8051 microcontroller. Whenever the LED blinks, it then gives an interrupt signal to the microcontroller of the 8051 family and thus the program of the microcontroller counts the pulses and displays the reading on the LCD duly interfaced to the microcontroller for every minute / daily / weekly or monthly as programmed which is sent to the cell phone of the user by an SMS through a GSM modem, which is interfaced to the microcontroller.
\end{abstract}

Keywords- Gsm module, nuvotonn microcontroller, electricity bill, energy meter, electricity

\subsection{Embedded Systems:}

\section{INTRODUCTION}

An embedded system is a combination of software and hardware to perform a dedicated task. Some of the main devices used in embedded products are microprocessors and micro-controllers. An embedded system is a computer system designed to perform one or more dedicated functions often with real-time computing constraints. It is embedded as a part of a complete device often including hardware and mechanical parts. By contrast ,a general-purpose computer ,such as personal computer (PC), is designed to be flexible and to meet a wide range of end-user needs. Embedded systems control many devices which are in common use today. The architecture of embedded system is as shown in figure.

\section{DESCRIPTION OF THE PROJECT}

The main aim of the Programmable energy meter project is to estimate energy consumed by load with the help of atmega and calculate the cost. Energy meter gives pulses which will be counted and displayed on LCD. These pulses occur depending on energy consumed. GSM to send SMS on amount of energy consumed. $12 \mathrm{~V}$ Transformer to give power supply to the system. The user needs to make call which will be received and displayed on LCD and stored. The system then comes to setup mode from where the user can switch to start or setting option, setting to set unit cost. Start to know the unit consumed and the cost. The system switches the loads ON/OFF Depending on user commands received through SMS. The system also includes a feature in which user can specify the number of days for which the user wants to retrieve the consumed and the estimated cost for the specified days.

In this design, the hardware components that we use are:

- GSM MODULE

- TRANSFORMER

- ULN2003A (RELAY DRIVER)

- Relay

- ENERGY METER

- OPTO COUPLER

\subsection{GSM MODULE}

A GSM modem duly interfaced to the MC through the level shifter IC Max232. The SIM card mounted GSM modem upon receiving digit command by SMS from any cell phone send that data to the MC through serial communication. While the program is executed, the GSM modem receives command 'STOP' to develop an output at the MC, the contact point of which are used to disable the ignition switch. The command so sent by the user is based on an intimation received by him through the GSM modem 'ALERT' a programmed message only if the input is driven low. The complete operation is displayed over $16 \times 2$ LCD display.

\subsection{POWER SUPPLY(TRANSFORMER):}

LPC2148 works on $3.3 \mathrm{~V}$ power supply. LM 117 can be used for generating $3.3 \mathrm{~V}$ supply. However, basic peripherals like LCD, ULN 2003 (Motor Driver IC) etc. works on 5V. So AC mains supply is converted into $5 \mathrm{~V}$ using below mentioned circuit and after that LM 117 is used to convert $5 \mathrm{~V}$ into $3.3 \mathrm{~V}$. It is used to step down $230 \mathrm{~V}$ AC to $9 \mathrm{~V}$ AC supply and provides isolation between power grids and circuit.

\section{SS2.3 RELAY}

A relay is usually an electromechanical device that is actuated by an electrical current. The current flowing in one circuit causes the opening or closing of another circuit. Relays are like remote-control switches and are used in many applications because of their relative simplicity, long life, and proven high reliability. Relays are used in a wide variety of applications throughout industry, such as in telephone exchanges, digital computers and automation systems. Highly 
sophisticated relays are utilized to protect electric power systems against trouble and power blackouts as well as to regulate and control the generation and distribution of power.

\subsection{RELAY DRIVER (ULN2003A)}

The ULN2003 is a monolithic high voltage and high current Darlington transistor arrays. It consists of seven NPN darlington pairs that features high-voltage outputs with common cathode clamp diode for switching inductive loads. The Darlington pairs may be paralleled for higher current capability. Each channel rated at $500 \mathrm{~mA}$ and can withstand peak currents of $600 \mathrm{~mA}$.

\section{SOFTWARE REQUIRED}

\subsection{KEIL Micro vision IDE for Programming:}

Compilers are programs used to convert a High Level Language to object code. Desktop compilers produce an output object code for the underlying microprocessor, but not for other microprocessors. I.E the programs written in one of the HLL like ' $C$ ' will compile the code to run on the system for a particular processor like $\mathrm{x} 86$ (underlying microprocessor in the computer). For example compilers for Dos platform is different from the Compilers for UNIX platform. The advantage of interpreters is that they can execute a program immediately. Secondly programs produced by compilers run much faster than the same programs executed by an interpreter. However compilers require some time before an executable program emerges. Now as compilers translate source code into object code, which is unique for each type of computer, many compilers are available for the same language.

\subsection{PROTEUS:}

The Proteus is an electronic circuit design software which includes a schematic capture, simulation and PCB ( Printed Circuit Board) Layout modules. But generally now a days Eagle CAD is highly preferred over Proteus for PCB designing because of its flexibility. Even though if $u$ are not using for PCB designing u can view the PCB layout of the component individually while selecting the component it helps during the soldering of components in PCB. Proteus is ahead in simulating the circuits containing the micro controllers where we can simulate the circuit by uploading the hex code to the Micro-controller where as Multisim can't do this

\section{DESIGN OF THE SYSTEM}

The Embedded system designed is Programmable Energy Meter With Bill Estimation using GSM module. This Project is designed with latest and most demanding technology that is embedded system. In this project we will use GSM based programmable energy meter to solve statistical errors overcome in the process of monthly billing. This idea of wireless data transmission is being planned to decrease the human involvement to gather the monthly reading and to minimize the technical problems about the billing process. This will help in a huge reduction of power thefts as well to analyze average power consumption of a certain locality. An automatic meter reading system is an actual way of data collection, that permit substantial saving through the saving of meter read ,more accuracy, allow regular reading, better billing, condensed tempering.

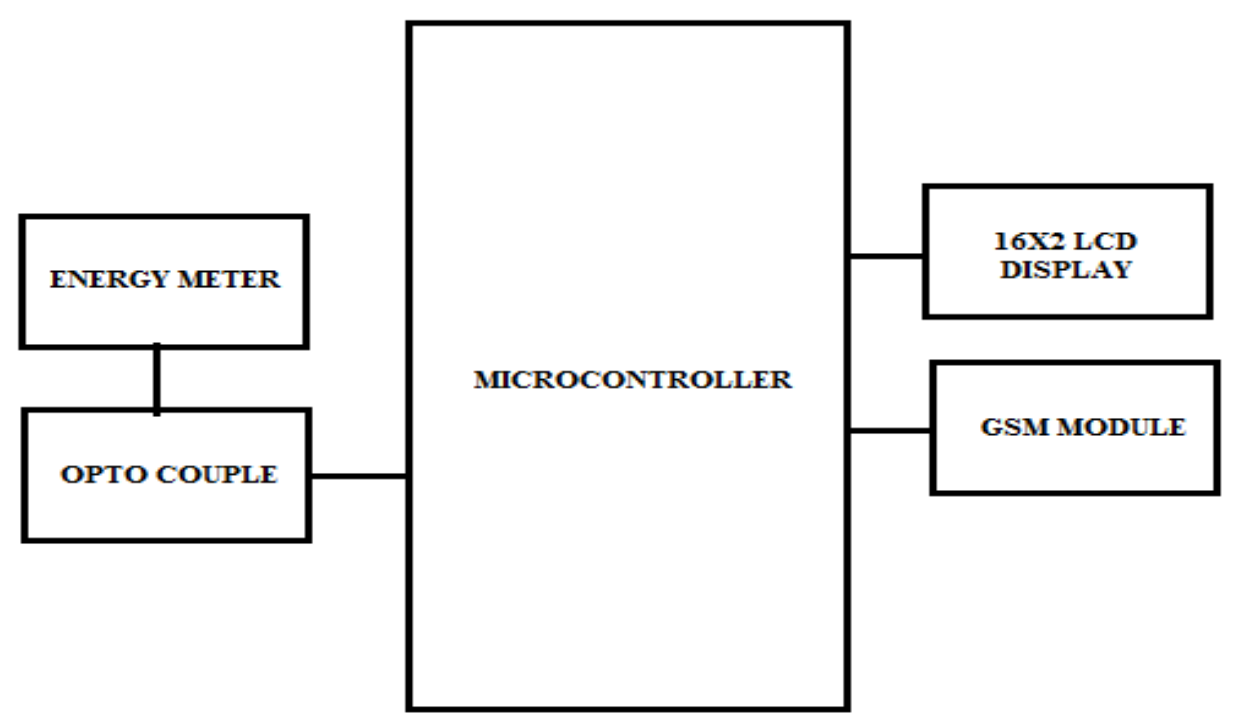

Fig. 1 Block diagram of the system 
In this design, the hardware components that we use are:

- ENERGY METER

- OPTO COUPLER

- ULN2003A

- Relay switch

- LCD DISPLAY

- MICRO CONTOLLER

- GSM MODULE

- BULB

LPC2148 works on $3.3 \mathrm{~V}$ power supply. LM 117 can be used for generating $3.3 \mathrm{~V}$ supply. However, basic peripherals like LCD, ULN 2003 (Motor Driver IC) etc. works on 5V. So AC mains supply is converted into $5 \mathrm{~V}$ using below mentioned circuit and after that LM 117 is used to convert $5 \mathrm{~V}$ into $3.3 \mathrm{~V}$. The port acting as the output to the microcontroller are connected to the input ports of ULN2003 IC. The output of ULN is connected Microcontroller the gsm module acts as a wireless data transmission between the micro controller and other peripherals. In gsm module, we are using transmitter pin, ground, Vcc pin. The ground pin is connected to the GND (pin 20) of the microcontroller thereby sharing a common ground. The Vcc pin of gsm module is connected to the PCB in which the voltage regulator7812 regulates $12 \mathrm{v} / 7805$ regulates $5 \mathrm{~V}$ to gsm module and the transmitter is connected the port 2.2 and receiver is connected to the port 2.1 which acts input from gsm module to the microcontroller. We use Nuvoton because it has an In System Programming (ISP). ISP is programming the microcontroller while the chip is on board and connected to many other components. During ISP every pin on microcontroller is disabled and chip is programmed by means of a programmer. But we write the programming for Atmel AT89C51 in keil micro software and we draw the circuit diagram of the system in proteus software. The proteus software is primarily for electronic design automation. The program on how to work the system and the circuit diagram of the system is burned into the microcontroller using the Nuvoton ISP-ICP utility.

\section{SCHEMATIC DIAGRAM:}

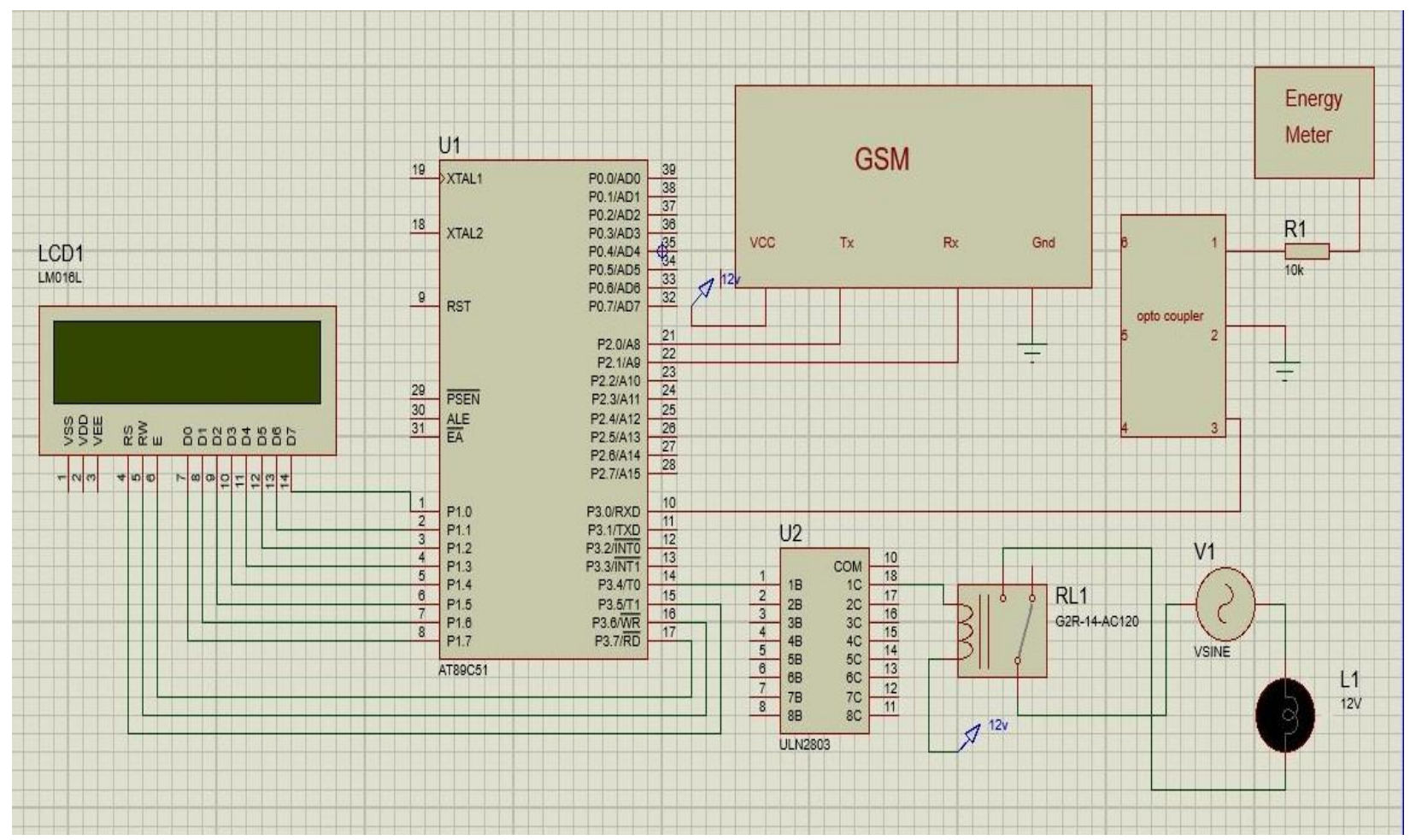

Fig. 2 Schematic diagram 


\section{RESULT}

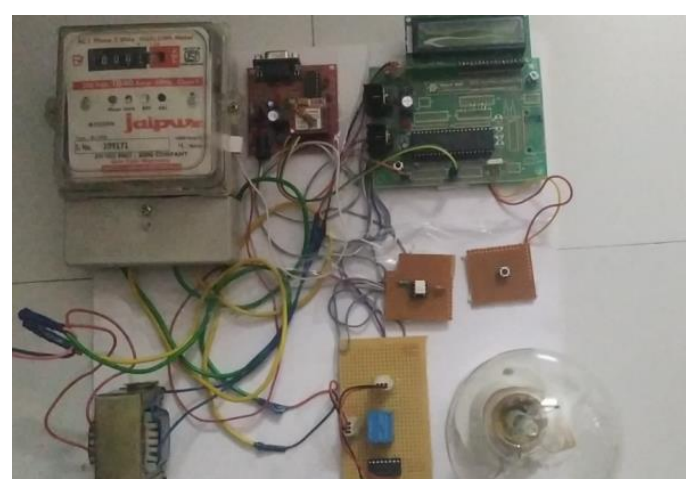

ADVANTAGES AND DISADVANTAGES

Advantages

1. This reduces the large number of labour operators/employees and long hours to accomplish the task and errors done by humans.

2. Less time consuming, Economical and simple to use.

3. This is going to help the customers to reduce their power bill by managing their energy consumption.

\section{Disadvantages}

1. Network Problem.

2. Circuit Complexity.

3. Implementation cost is high.

Applications :

\section{APPLICATIONS}

1.It is used wherever electricity is needed.

Eg: Industries, Houses

\section{CONCLUSION}

GSM is beneficial for both energy provider and customer. This reduces the manual cost and also reduces the errors done by the humans. The statistical load use and profile help the customer to manage their energy consumption. This helps the consumer to reduce the number of units. This system can be used even in the remote areas by changing the type of the modem and its range of frequency for communication. This device reduces all the cases of revenue problems to the country and helps us to improve our usage.

\section{FUTURE SCOPE}

In future we can also update the tariff within the energy meter by writing a program in the java and it must be connected to the energy meter using USB port which automatically updates the program in the microcontroller. And we must also make easy to the customers for buying the watts using SMS request. This makes flexible for both user and the company.

\section{REFERENCE}

[1] H.G. Rodney Tan, C.H. Lee, and V.H. Mok Design and Implementation of Automatic Meter Reading System Using GSM, ZIGBEE through GPRS, International Journal of Advanced Research

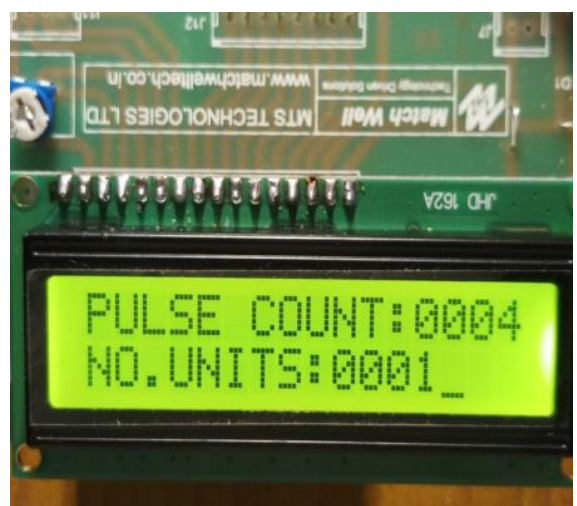

in Computer Science and Software Engineering,vol2, edition 5, May 2012.

[2] Yujun Bao and Xiaoyan Jiang, "Design of electric Energy Meter for long-distance data information transfers based upon GPRS", ISA 2009. International Workshop on Intelligent Systems and Applications, 2009.

[3] H.G. Rodney Tan, C. H. Lee, and V. H. Mok, "Auto- matic Power Reading Using GSM Network", The 8th International Power Engineering Conference (IPEC2007).

[4] Subhashis Maitra, "Embedded Energy Meter- A new concept to measure the energy consumed by a con- sumer and to pay the bill", Power System Technology and IEEE Power India Conference, 2008

\section{WEBSITES VIEWED}

http://www.slideshare.net/ikm104/gsm-energy-meter www.keil.com

\#include <reg51.h>

\section{APPENDIX}

sbit $\mathrm{rs}=\mathrm{P} 3^{\wedge} 5$;

sbit $\mathrm{rw}=\mathrm{P} 3^{\wedge} 6$;

sbit en $=\mathrm{P} 3 \wedge$;

sbit $\mathrm{sw}=\mathrm{P} 1^{\wedge} 6$;

sbit $\mathrm{r} 1=\mathrm{P} 1^{\wedge} 1$;

sbit sen $=\mathrm{P} 1^{\wedge} 7$;

void gsm(void);

void sense(void);

unsigned int bill $=0$, count $=0, \mathrm{u}=0$, count $1, \mathrm{u} 1$, bill1 1 , unit;

float watt_factor $=0.3125$;

void delay(unsigned int $\mathrm{x}$ )

\{

unsigned int $\mathrm{i}$;

for $(; \mathrm{x}>0 ; \mathrm{x}--)$

for $(i=0 ; i<225 ; i++)$;

\}

unsigned char $\mathrm{rx}()$

\{

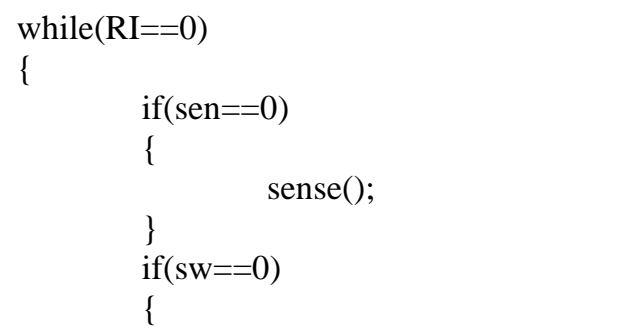




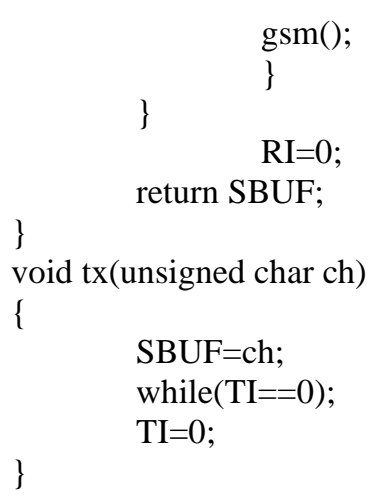

void disp_str(unsigned char ${ }^{*} \mathrm{x}$ )

\{

while $(* x)$

\{

\}

$$
\operatorname{disp}\left({ }^{*} \mathrm{x}++\right)
$$

\}

void lcd_val(unsigned int val)

\{

unsigned char a1,a2,a3,a4;

a1 $=($ val\% 10000 $) / 1000+48$;

a2 $=($ val\% 1000 $) / 100+48$;

a3 $=($ val\% 100) $/ 10+48$;

$\mathrm{a} 4=(\mathrm{val} \% 10)+48$;

disp(a1);

$\operatorname{disp}(\mathrm{a} 2)$;

disp(a3);

$\operatorname{disp}(\mathrm{a} 4)$;

\}

void main()

\{

unsigned char $\mathrm{i}=0, \mathrm{ch}=0, \mathrm{n}=0, \mathrm{j}=0, \operatorname{dum}[10], \mathrm{c}=0$; unsigned char num[15];

$\operatorname{lcdinit}()$;

$\mathrm{SCON}=0 \times 50$;

TMOD $=0 \times 20$;

TH1=-3;

TR1 1 ;

cmd(0x01); delay(500);

cmd(0x80);

$\mathrm{r} 1=0$;

disp_str("GSM BASED ENERGY ");

cmd(0xc0);

disp_str("METER READING ...."); delay(2000);

cmd $(0 x 01)$; cmd $(0 x 80)$;

disp_str("AT");

uart_str("AT");

$\operatorname{tx}(0 \mathrm{x} 0 \mathrm{~A})$;

$\operatorname{tx}(0 \mathrm{x} 0 \mathrm{D})$;

delay(1000);

cmd(0x01);

cmd $(0 \times 80)$;

disp_str("AT+CMGF=1"); uart_str("AT+CMGF=1"); $\operatorname{tx}(0 \mathrm{x} 0 \mathrm{~A})$; $\operatorname{tx}(0 \mathrm{x} 0 \mathrm{D})$; delay(1000); uart_str("AT+CMGD=");

$\operatorname{tx}(' 1 ')$;

$\operatorname{tx}(0 \mathrm{x} 0 \mathrm{~A})$;

$\operatorname{tx}(0 \mathrm{x} 0 \mathrm{D})$;

uart_str("AT+CMGD=");

tx('2');

tx $(0 \mathrm{x} 0 \mathrm{~A})$;

$\operatorname{tx}(0 \mathrm{x} 0 \mathrm{D})$; 


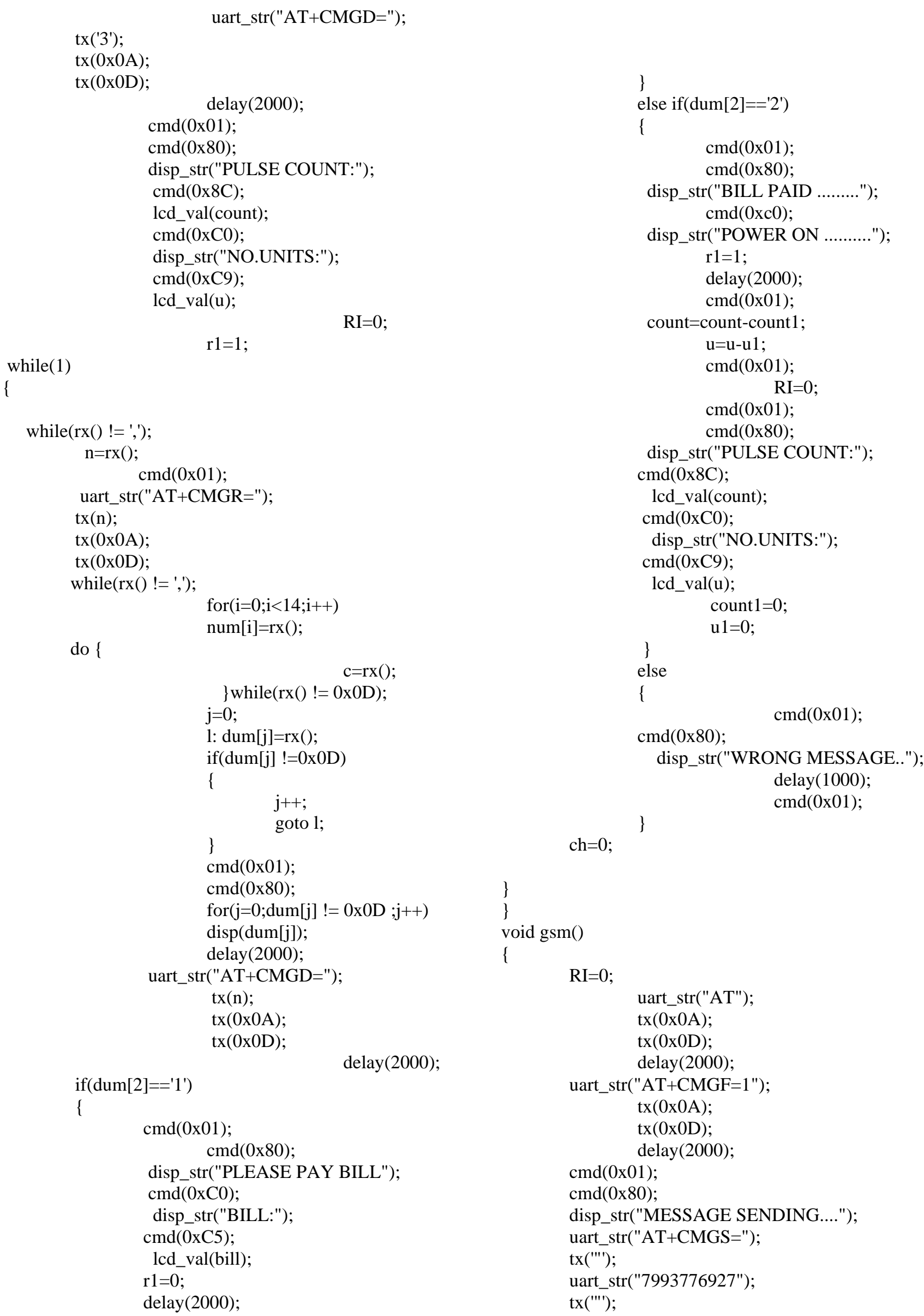

$\operatorname{tx}\left({ }^{\prime} 3^{\prime}\right)$;

$\mathrm{tx}(0 \mathrm{x} 0 \mathrm{~A})$;

$\operatorname{tx}(0 \mathrm{x} 0 \mathrm{D})$;

cmd $(0 x 01)$

$\operatorname{delay}(2000)$;

cmd $(0 \times 80)$;

disp_str("PULSE COUNT:");

cmd(0x8C);

lcd_val(count);

cmd $(0 \mathrm{xC} 0)$;

disp_str("NO.UNITS:");

cmd(0xC9);

lcd_val(u);

$\mathrm{RI}=0$;

while(1)

$$
\mathrm{r} 1=1 \text {; }
$$

\{

do \{ num $[\mathrm{i}]=\mathrm{rx}()$;

\} else if(dum[2]=='2')

\{

disp_str("PULSE COUNT:"); cmd $(0 \mathrm{x} 8 \mathrm{C})$;

lcd_val(count);

cmd $(0 \mathrm{xC} 0)$;

disp_str("NO.UNITS:");

cmd(0xC9);

lcd_val(u); count $1=0$; $\mathrm{u} 1=0$;

\}

else

\{

cmd(0x80);

$\operatorname{cmd}(0 \mathrm{x} 01)$

disp_str("WRONG MESSAGE.."); delay(1000); cmd(0x01);

ch=0;

\}

void gsm()

\{ 
$\operatorname{tx}(0 \mathrm{x} 0 \mathrm{~A})$

$\operatorname{tx}(0 \mathrm{x} 0 \mathrm{D})$;

delay(2000);

uart_str("MONTH COMPLETED PLEASE PAY

THE BILL AMOUNT AND UNITS IS:");

$\operatorname{tx}(0 \mathrm{x} 0 \mathrm{~A})$;

tx $(0 \mathrm{x} 0 \mathrm{D})$;

convert(u); uart_str("UNITS:");

$\operatorname{tx}(0 \mathrm{x} 0 \mathrm{~A})$;

tx(0x0D);

uart_str("RS:");

bill $=\mathrm{u} * 5$;

convert(bill);

delay(2000);

cmd (0x01);

$\operatorname{tx}(0 \mathrm{x} 1 \mathrm{~A})$;

delay(3000);

uart_str("AT");

$\operatorname{tx}(0 \mathrm{x} 0 \mathrm{~A})$;

$\operatorname{tx}(0 \mathrm{x} 0 \mathrm{D})$;

delay(2000);

uart_str("AT+CMGF=1");

$\operatorname{tx}(0 \mathrm{x} 0 \mathrm{~A})$;

$\operatorname{tx}(0 \mathrm{x} 0 \mathrm{D})$;

delay(2000);

cmd $(0 \mathrm{x} 01)$;

cmd(0x80);

disp_str("MESSAGE SENDING....");

uart_str("AT+CMGS=");

tx("'");

uart_str("7013684692");

tx('"');

$\operatorname{tx}(0 \mathrm{x} 0 \mathrm{~A})$;

$\operatorname{tx}(0 \mathrm{x} 0 \mathrm{D})$;

delay(2000);

uart_str("MONTH COMPLETED PLEASE PAY

THE BILL AMOUNT AND UNITS IS:");

$\operatorname{tx}(0 \mathrm{x} 0 \mathrm{~A})$;

$\mathrm{tx}(0 \mathrm{x} 0 \mathrm{D})$;

uart_str("UNITS:");

convert(u);

tx $(0 \mathrm{x} 0 \mathrm{~A})$;

$\operatorname{tx}(0 \mathrm{x} 0 \mathrm{D})$;

uart_str("RS:");

bill $=\mathrm{u} * 5$;

convert(bill);

delay(2000);

cmd(0x01);

tx $(0 \mathrm{x} 1 \mathrm{~A})$;

bill1=bill;

delay(3000);

count $1=$ count;

$\mathrm{u} 1=\mathrm{u}$;

delay(1000);

$$
\begin{gathered}
\text { RI=0; } \\
\text { cmd(0x80); } \\
\text { disp_str("MONTH COMPLETED...."); } \\
\text { cmd(0xc0); } \\
\text { disp_str("PLEASE PAY BILL " "); }
\end{gathered}
$$

cmd(0x01);

cmd(0x80);

disp_str("PULSE COUNT:"); cmd(0x8C);

lcd_val(count);

cmd $(0 \times \mathrm{C} 0)$;

disp_str("NO.UNITS:");

cmd(0xC9);

lcd_val(u); delay(1000);

\}

void sense()

\{

count++;

cmd(0x8C);

lcd_val(count);

$\mathrm{u}=$ watt_factor*count $/ 1$; cmd(0xC9);

delay(200); lcd_val(u); 